\title{
Prospecção de Cenários para o período de 2019 a 2030 dos Programas de Stricto Sensu das Instituições do Brasil da Área de Administração Pública e de Empresas, Ciências Contábeis e Turismo
}

\author{
Henrique César Melo Ribeiro ${ }^{1}$ \\ Sergio Henrique Arruda Cavalcante Forte ${ }^{2}$
}

\section{Resumo}

O objetivo deste estudo foi elaborar os Cenários Prospectados para o período de 2019 a 2030 dos Programas de Stricto Sensu das Instituições do Brasil da Área de Administração Pública e de Empresas, Ciências Contábeis e Turismo. Utilizou-se a metodologia de cenários sugerida por Blanning e Reinig. Os resultados apontam 14 eventos. No cenário otimista estes eventos vislumbram a importância que os programas terão para com o corpo docente e as parcerias, contribuindo para pesquisas de qualidade. Já no cenário realista os eventos dão ênfase aos docentes, mas sobretudo às estratégias competitivas, como por exemplo a internacionalização. No cenário pessimista os eventos discorrem sobre o processo seletivo e os egressos dos programas. Este estudo, traz em seu bojo as nuances de eventos que se relacionam a cenários prospectados para o período de 2019 a 2030 dos programas stricto sensu do Brasil, contribuindo para o avanço do conhecimento, e, consequentemente, colaborando para o fomento do meio científico, impactando no desenvolvimento social e econômico e, posteriormente cooperando para que estes programas evoluam na vitrine do mercado acadêmico nacional e quiçá internacional.

Palavras-chave: Prospeç̧ão de cenários; Programas de stricto sensu do Brasil; Área de Administração Pública e de Empresas, Ciências Contábeis e Turismo.

\section{Prospecting Scenarios for the period from 2019 to 2030 of the Stricto Sensu Programs of the Brazilian Institutions of Public Administration and Business, Accounting and Tourism}

\begin{abstract}
The objective of this study was to elaborate the Prospected Scenarios for the period from 2019 to 2030 of the Stricto Sensu Programs of the Brazilian Institutions of Public Administration and Business, Accounting and Tourism. We used the scenario methodology suggested by Blanning and Reinig. The results show 14 events. In the optimistic scenario these events see the importance that the programs will have for the faculty and the partnerships, contributing to quality research. In the realist scenario, the events emphasize the reseachers, but above all the competitive strategies, such as internationalization. In the pessimistic scenario the events talk about the selective process and the graduates of the programs. This study brings to the fore the nuances of events that relate to scenarios prospected for the period from 2019 to 2030 of the stricto sensu programs of Brazil, contributing to the advancement of knowledge, and, consequently, collaborating to foster the scientific, impacting on social and economic development, and subsequently cooperating so that these programs evolve in the showcase of the national and perhaps international academic market.
\end{abstract}

Keywords: Prospecting scenarios; Stricto sensu programs in Brazil; Area of Public Administration and of Companies, Accounting Sciences and Tourism.

\footnotetext{
${ }^{1}$ Universidade Federal da Piauí, Teresina (Brasil). E-mail: hొmribeira国gmail.com Drcid id: https://arcid.org/Q000-0002-0704-1812

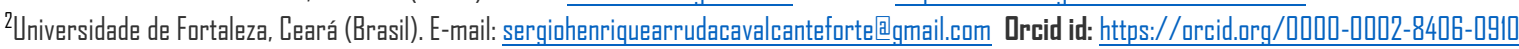




\section{INTRODUÇÃO}

O exercício de construir cenários para diferentes setores de atuação pode ser um dos mais estratégicos aspectos para a sobrevivência e competitividade no mercado (Faller \& Almeida, 2014). Diante desse contexto, nota-se que o papel da educação é importante para prospectar cenários alternativos (Blass, Thornton \& Rawlings, 2011; Wright \& Goodwin, 1999). Versa-se, assim, a importância de entender e compreender as condicionantes, tendências e a prospecção do cenário na educação (Alarcão, Tavares, Mealha \& Souza, 2018; Thiesen, Garcez \& Guimaraes, 2014), sobretudo no ensino superior (Silva, Machado Neto \& Santos, 2015) e mais especificamente na pós-graduação brasileira (Moritz, Moritz, Pereira \& Maccari, 2013).

A pesquisa de prospecção de cenários vem se tornando um instrumento valoroso para a educação superior (Rieckmann, 2012) nas Instituições de Ensino Superior (IESs) públicas e privadas do Brasil, pois contribui para a estratégia dos programas de pós-graduação, sobretudo para o planejamento estratégico (Lindgren \& Bandhold, 2003) alicerçado em uma investigação prospectiva de cenários/futuros prováveis/preferidos (Ithnin, Sahib, Eng, Sidek \& Harun, 2018), sendo possível, assim, influenciar positivamente no clima organizacional (Chermack, Coons, Nimon, Bradley \& Glick, 2015), na mitigação de riscos (Godet, 2000), facilitando o processo decisórios (Oliveira \& Forte, 2009), as tomadas de decisões e as escolhas de estratégias competitivas (Porter, 1980) que, porventura, serão implementadas e adotadas (Sobreira, Machado, Rebouças \& Forte, 2014).

Sousa, Forte e Oliveira (2012) identificaram as dimensões de recursos estratégicos utilizadas pelas IES privadas da Região Nordeste para um cenário mais provável de ocorrência no período 2009/2015. Os resultados demonstram que o Cenário "Tudo pelo Mercado Adaptado" é o mais provável e que as IESs estão valorizando recursos que não proporcionam diferencial competitivo.

Moritz et al. (2013) concluíram, em sua pesquisa, que é esperado pela pósgraduação para 2020 no Brasil, que ela aja com uma consciência social alargada, atenta ao ambiente para aprendizagens expressivas e aos novos tempos da humanidade, em que pesquisa e trabalho são variáveis que não se excluem, e os que nela atuam necessitam utilizar de suas competências para achar opções de modificação a partir de práticas inovadoras nos mestrados e doutorados, cujas 
diferentes demandas começam a colocar-se no presente, seja de fato relevante para um renovado e inovador Brasil do futuro.

Alarcão et al. (2018) construíram cenários prospectivos sobre a Universidade nas dimensões da formação, inovação e pesquisa e em função da intensidade valorativa. Os autores salientaram a oportunidade de melhor compreender um ambiente socioconstrutivo, refletindo sobre o que poderá ser a Universidade do futuro em uma visão a médio prazo e quais as implicações nas dimensões de formação, de inovação e de pesquisa.

Spada e Forte (2018) identificaram os cenários prospectados para o horizonte do ano de 2030 para as Universidades Corporativas no Brasil. Concluíram que a prospecção de cenários traz às instituições de ensino superior brasileiras bases mais sólidas para compor suas estratégias em médio e longo prazos.

Heinzen e Marinho (2019) propuseram um modelo para alinhava formulação e implementação da estratégia em IES. Ressalta-se que, para a construção do modelo, foi utilizado a metodologia de cenário como input e considerados os principais elementos que possibilitam a formulação e implementação da estratégia. Os autores concluíram que a metodologia de cenário é um instrumento relevante para a gestão estratégica das IES.

Diante do exposto, e considerando o foco desta pesquisa, após ser realizado um levantamento da análise de cenários relacionados à área de educação, mas propriamente da pós-graduação, em especial do stricto sensu, constatou-se que poucos estudos investigaram a aplicação e o uso dos métodos de cenários no setor da educação superior (Heinzen, 2015).

Em vista disso, versa-se a seguir a questão de pesquisa que orientou e norteou este estudo que foi: Quais os Cenários Prospectados para o período de 2019 a 2030 dos Programas de Stricto Sensu das Instituições do Brasil da Área de Administração Pública e de Empresas, Ciências Contábeis e Turismo? O objetivo foi elaborar os Cenários Prospectados para o período de 2019 a 2030 dos Programas de Stricto Sensu das Instituições do Brasil da Área de Administração Pública e de Empresas, Ciências Contábeis e Turismo.

Reforça-se a justifica de se realizar esta pesquisa por ter sido constatado que o número de estudos sobre cenários ainda ser muito pequeno na literatura acadêmica nacional (Carlos, Paiva Filho \& Forte, 2002) e também internacional 
(Pereira, Barros, Oliveira, Gomes \& Costa, 2015), e, portanto, existe um espaço macro para a pesquisa acadêmica sobre o citado tema. Ademais, pesquisas futuras são importantes e necessárias para, por exemplo, se identificar como vem ocorrendo a utilização dos processos e métodos de prospecção de cenários nas organizações (Moritz, Nuner \& Pereira, 2008).

Outra justificativa para a concepção deste estudo que é o ambiente de intenso debate quanto ao papel e os escopos da pós-graduação no Brasil e da qualidade de suas propostas acadêmicas, em um instante em que se demandam programas de pós-graduação que respondam à grande variedade de desafios sociais, tecnológicos, políticos e ecológicos. Nesse contexto, é de grande importância e relevância refletir sobre as condições e contribuições atuais e o cenário futuro desejável para estas pós-graduações (Moritz et al., 2013), em especial o stricto sensu.

Acredita-se que este estudo poderá suprir as demandas de um cenário melhor para os programas stricto sensu das IESs públicas e privadas do Brasil da área de Administração Pública e de Empresas, Ciências Contábeis e Turismo mediante a oferta das metodologias e das ferramentas que a literatura disponibiliza, além da identificação e realce dos eventos-chaves que possam ter elevado impacto na mencionada área do conhecimento.

A escolha temporal de 12 anos para este estudo se justifica em razão, primeiramente porque a horizontalidade de tempo do cenário, em média, pode ser de dez anos (Silva, 2016), ou seja, é admissível se trabalhar com uma projeção inferior (Oliveira \& Forte, 2011), igual (Silva, 2010) ou superior a dez anos (Sobreira et al., 2014), pois o objetivo norteador da prospecção de cenários é de assessorar na definição de estratégias para a organização no longo prazo (Oliveira \& Forte, 2008). E a segunda justificativa é que 12 anos abrange o período de três governos do Brasil (2019-2022, 2023-2026 e 2027-2030).

Como resultado o estudo mostra de maneira ampla, uma visão à luz de 14 eventos classificados nos cenários (otimista, realista e pessimista) organizados e prospectados para os programas stricto sensu objeto da investigação. Estes cenários com seus respectivos eventos realçados dão um norte e embasamento a estes programas de como procederem em cenários adversos (Figueiredo, Chedid \& Machado Neto, 2010), influenciando a posteriori em uma maior competitividade 
(Heinzen \& Marinho, 2019), colaborando e impactando em melhores níveis de desempenho empresarial (Fernandes, Fleury \& Mills, 2006).

Este estudo divide-se em cinco seções. A primeira é a Introdução. Logo em seguida, versa-se o Referencial Teórico do estudo. Os Procedimentos Metodológicos são evidenciados na seção três. Na quarta seção são feitas as Análises e Discussões dos Resultados. E, por fim, na seção cinco são contemplados os resultados, conclusões e contribuições do estudo, como também a limitação e sugestões para pesquisas futuras.

\section{REFERENCIAL TEÓRICO}

Esta seção versa as subseções prospecção e cenários: estudos; e pósgraduação no Brasil.

\subsection{PROSPECÇÃO E CENÁRIOS}

Prospecção remete à estratégia (Godet, 2010) e essa estratégia é baseada em cenários (Moorcroft, 2015). Diante disso, entende-se que o planejamento de cenários é uma ferramenta de cenários que é amplamente utilizado no planejamento estratégico (Bowman, 2016; Tapinos, 2013). Neste panorama, versa-se que a atitude prospectiva não espera pela alteração e depois reage, ela visa dominar a mudança esperada (preatividade) e induzir uma transformação desejada (proatividade). Preatividade é o que orienta todas as abordagens para futuros estudos, previsão, planejamento de cenários e prospecção. A proatividade é mais voluntarista, e visa trazer as modificações desejadas por meio do planejamento estratégico. O que se força a pensar: é importante repensar os problemas para avançar (Godet, 2010).

No entanto, um elemento subdesenvolvido do planejamento de cenários é a avaliação de estratégias alternativas em toda a gama de cenários. Se isso for feito de maneira informal, então estratégias inferiores podem ser selecionadas, enquanto os procedimentos de avaliação formal, que foram sugeridos em relação ao planejamento de cenários, provavelmente, não serão práticos na maioria dos contextos (Goodwin \& Wright, 2001). Com isso, o planejamento de cenários é visto como uma disciplina acadêmica (Derbyshire, 2016) de investigação que pode gerar 
pesquisas interessantes, inovadoras, utilizáveis, rigorosas, fornecendo, com isso, insumos para estudos futuros (Ramirez, Mukherjee, Vezzoli \& Kramer, 2015).

Diante do exposto, versa-se que existem muitos tipos diferentes de estudos futuros e estes podem ser perseguidos com objetivos diferentes. Este é o ponto de início para uma classificação que sugere que todos os estudos futuros respondem a uma das três perguntas: 'o que vai acontecer?', "o que pode acontecer?" e "como atingir um alvo específico?" (Höjer, Dreborg, Engström, Gunnarsson-Östling \& Svenfelt, 2011).

Neste contexto, ressalta-se que o estudo de cenários explora construções relacionadas ao pensamento de cenários, desenvolvimento de cenários, tais como ferramenta, estudos de cenários (Ramirez et al., 2015), além da prospecção de cenários e análise de cenários (Moritz, Nuner \& Pereira, 2008). Isto posto, observase que a análise de cenários oferece um excelente veículo de instrução para investigar 'problemas perversos', questões complexas e ambíguas e que exigem um inquérito transdisciplinar (Bradfield, Cairns \& Wright, 2015).

Diante disso, realça-se que a estratégia de cenários vem tomando lugar de extrema relevância e importância entre as ferramentas de suporte e melhora ao processo de tomada de decisão nas organizações (Wright \& Goodwin, 2009), e um exemplo disso é que, nas últimas décadas, a literatura acadêmica sobre cenários registrou crescimento considerável nas publicações em revistas internacionais (Amer, Daim \& Jetter, 2013; Oliveira, Barros, Pereira, Gomes \& Costa, 2018; Varum \& Melo, 2010) e nacionais (Weiss, Kremer, Trentin \& Rojo, 2017).

Entende-se e compreende-se que a utilização desta estratégia permite ao gestor, agir com mais segurança e melhor conhecimento da incerteza presente no futuro do cenário organizacional (Grant, 2003), garantindo, no processo decisório, que as decisões tomadas sejam com mais qualidade, criatividade, segurança e, consequentemente, velocidade (Moritz \& Pereira, 2005).

Cenário é um conjunto constituído pela redação minuciosa de uma perspectiva futura, incluindo a iniciativa dos principais atores e o percentual estimado de fronteiras incertas (Vecchiato, 2015), articuladas de tal maneira a descrever a passagem da ocorrência de origem para uma circunstância em um momento futuro de forma lógica (Godet, 1993). Assim sendo, "[...]com base na metodologia de cenários prospectivos, podem-se criar as estratégias que irão 
facilitar e agilizar os caminhos futuros do ambiente ou das organizações" (Moritz et al., 2013, p. 23).

Corroboram com esta afirmação, complementando-a os autores Blois e Souza (2008), ao evidenciarem que os cenários proporcionam um ambiente que enriquece o debate sobre perguntas críticas relacionadas ao futuro da empresa, permitindo que os gestores dessas organizações tomem decisões de risco com mais clareza e segurança, consentindo, ainda, a identificação de oportunidades e de ameaças ao negócio, promovendo o aprimoramento e a análise de novas opções perante as mudanças no contexto externo (Blois \& Souza, 2008).

Diante do exposto, pode-se constatar que construir cenários futuros, a fim de promover uma "visão de futuro" tão importante e necessária, a elaboração de estratégias competitivas, pode ser considerada como uma ação já aceita, utilizada e legitimada pelas melhores companhias do ambiente empresarial (Moritz, Nuner \& Pereira, 2008).

\subsection{PÓS-GRADUAÇÃO NO BRASIL}

De acordo com o parecer $n^{\circ}$. 977/1965, a pós-graduação stricto sensu no Brasil é categorizada em dois níveis: mestrado e doutorado (Paixão \& Bruni, 2013). Reforça-se que o objetivo do doutorado é o de aperfeiçoar a capacidade extensa de pesquisa; para tanto, o candidato deverá defender uma tese que proporcione uma real contribuição para o conhecimento científico de um determinado tema; e para o mestrado, exige-se a defesa de uma dissertação, que deve conceber a capacidade do candidato de sistematizar e dominar uma determinada temática (Paixão \& Bruni, 2013).

Foi implantada, pela primeira vez, a modalidade de pós-graduação stricto sensu no Brasil em 1967 (Cirani, Silva \& Campanario, 2012), com o objetivo de contribuir para o aprimoramento de cursos de doutorado e mestrado mediante a pesquisa científica e tecnológica (Maccari, Almeida, Mnishimura \& Rodrigues, 2009), sendo considerado um dos mais modernos do mundo (Maccari \& Lima; Riccio, 2009). Sua organização se deu por meio de esforços governamentais federais levando assim à criação de programas de mestrado e doutorado em universidades públicas e privadas. Versa-se que a pós-graduação stricto sensu é 
vista como um sucesso em termos de nações emergentes, ficando somente abaixo das encontradas nos países desenvolvidos (Bertero, Caldas \& Wood Jr, 1999).

No Brasil, o processo de avaliação da pós-graduação stricto sensu é conduzido pela Coordenação de Aperfeiçoamento de Pessoal de Nível Superior CAPES (Crespi, Preusler, Luna \& Ferreira, 2017; Patrus, Shigaki \& Dantas, 2018), sendo esta vinculada ao Ministério da Educação - MEC (CAPES, 2019; Maccari, Riccio \& Martins, 2013).

Ressalta-se que, além da CAPES conduzir todo o sistema de avaliação de cursos de pós-graduação stricto sensu, ela também avalia as propostas de cursos novos de pós-graduação no Brasil (CAPES, 2018; Moro, Balsan, Costa \& Schetinger, 2014). A área de Administração Pública, Administração de Empresas, Ciências Contábeis e Turismo agrupa 184 Programas de Pós-Graduação, 62 Doutorados, 107 Mestrados Acadêmicos e 75 Mestrados Profissionais. São 11 Programas de Pós-Graduação em Administração Pública, 135 Administração de Empresas, 27 Ciências Contábeis e 11 em Turismo (CAPES, 2016).

Entende-se que o Brasil está ainda aquém de um sistema de ensino capaz de amparar o surto de inovação necessário para posicionar-se no mercado global como algo mais que um grande exportador de commodities (Moritz et al., 2013). O aperfeiçoamento da pós-graduação impacta no crescimento deste sistema de inovação, influenciando, posteriormente, também na graduação com a inclusão de disciplinas e metodologias inovadoras, para que seja desenvolvido um novo profissional com competências, capacidades, habilidades e conhecedor de seu papel na sociedade, mas, sobretudo, envolvido com a pesquisa e com maior visão na tomada de decisão (Moritz et al., 2013).

Neste contexto, ressalta-se que as universidades, governo e sociedade podem e devem empregar a metodologia de cenários, pois ela ampara essas instituições, identificando seus principais eventos sociais e econômicos futuros, preparando-as para as várias probabilidades de acontecimentos futuros, dandoIhes condições para decisão mais estratégicas e proativas, com maior eficácia e efetividade e, logo, tornando-as propensas à implementação e formulação de um planejamento estratégico apropriado, harmônico, flexível e com a imprescindível visão de prospecção de cenários futuros (Moritz et al., 2013). 


\section{PROCEDIMENTOS METODOLÓGICOS}

Com o objetivo de elaborar os Cenários Prospectados para o período de 2019 a 2030 dos Programas de Stricto Sensu das Instituições do Brasil da Área de Administração Pública e de Empresas, Ciências Contábeis e Turismo. Este estudo prospectou os pareceres de gestores dos Programas de Stricto Sensu, embasada pela metodologia sugerida por Blanning e Reinig (1998). Justifica-se o uso da referida metodologia em virtude desta classificar os cenários em três tipos: otimista, pessimista e realista (Silva \& Forte, 2016).

O Método de Robert W. Blanning e Bruce A. Reinig foi desenvolvido em 1998 e tem a seguintes macroetapas para elaboração dos Cenários: (i) consultar um grupo de peritos; (ii) os resultados são expostos aos especialistas; (iii) ocorre a construção da lista de eventos; (iv) repete-se o procedimento, para que sejam indicados a probabilidade de ocorrência dos eventos listados, numa escala de zero a dez; ( $v$ ) averiguar com os especialistas, a partir das respostas dadas em relação às probabilidades de frequência dos eventos, se serão aderentes ou adversas a organização foco da pesquisa; (vi) construir uma matriz de eventos, colocando no eixo horizontal as probabilidades de ocorrência destes eventos $(P)$ e, no eixo vertical, o quanto cada evento é aderente ou contrário $(F)$ para a organização que está desenvolvendo a análise; e (vii) propor a construção de três cenários: otimista, realista e pessimista (Heinzen, 2015; Heinzen \& Marinho, 2019).

Entende-se, com isso, que, essa investigação é o tipo exploratória e descritiva. Versa-se que a pesquisa exploratória é um meio de acender ideias, maximizar a afinidade do pesquisador com o tema em análise e elucidar teorias. Assim, para se alcançar o objetivo proposto para este estudo, foi trabalhada uma pesquisa de escopo exploratório, dividida em uma fase qualitativa e outra quantitativa (Espinoza \& Hirano, 2003).

Portanto, justifica-se este estudo como exploratório, pois se efetivou um levantamento do marco teórico que embasa este trabalho, além da realização das entrevistas exploratórias com peritos (pesquisadores da área), para interpretar a realidade a partir dos dados coletados, com o foco de identificar variáveis chaves para a construção de uma lista de eventos (Brock \& Barry, 2003) e, consequentemente, para prospectar cenários sobre o tema ora investigado. 
Em relação a esta pesquisa ser descritiva, é em decorrência de sua busca para a resolução de problemas mediante entrevistas com especialistas para validação de conteúdo, aperfeiçoando os aprendizados, seja pela verificação, análise e exposições práticas (Thomas, Nelson \& Silverman, 2007). Em suma, a pesquisa descritiva investiga e correlaciona variáveis (fenômenos/fatos) sem manipulá-los (Erfurth \& Bezerra, 2013), usando padrões textuais como, por exemplo, questionários para identificação do conhecimento (Malhotra et al., 2005), contribuindo para o desenvolvimento dos três cenários prospectados: (i) cenário otimista; (ii) cenário realista; e (iii) cenário pessimista (Blanning \& Reinig, 1998).

O universo deste estudo foram os Programas Stricto Sensu das Instituições de Ensino Superior Públicas e Privadas do Brasil da Área de Administração Pública e de Empresas, Ciências Contábeis e Turismo. Em razão de este universo contribuir para o aperfeiçoamento de cursos de doutorado e mestrado por meio do trabalho científico (Maccari et al., 2009); e se verificar que a pós-graduação stricto sensu é um sucesso em termos de nações emergente (Bertero, Caldas \& Wood Jr, 1999), justifica-se, assim, focar este estudo neste universo de pesquisa.

Evidenciam-se que os Programas Stricto Sensu das Instituições de Ensino Superior Públicas e Privadas do Brasil da Área de Administração Pública e de Empresas, Ciências Contábeis e Turismo existentes e associados a Associação Nacional de Pós-Graduação e Pesquisa em Administração (ANPAD) fizeram parte da população do estudo desta pesquisa. Versa-se que a amostra dos Programas Stricto Sensu das Instituições de Ensino Superior Públicas e Privadas do Brasil da Área de Administração para se realizar este estudo foi feita por acessibilidade, composta pelos Programas Associados a ANPAD, composto por 126 programas.

A primeira etapa de coleta de dados da referida pesquisa, foram as entrevistas com cinco peritos (Quadro 1), todos doutores e vinculados a programas de pós-graduação stricto sensu da área de Administração Pública e de Empresas, Ciências Contábeis e Turismo.

\begin{tabular}{|c|c|c|}
\hline Peritos & Organização & Curso/Programa \\
\hline Entrevistado 1 & IES privada & Programa de Mestrado Profissional em Administração \\
\hline Entrevistado 2 & IES privada & $\begin{array}{c}\text { Doutorado e Mestrado em Administração } \\
\text { Programa de Mestrado }\end{array}$ \\
\hline Entrevistado 3 & IES privada & Programa de Mestrado Acadêmico em Administração \\
\hline Entrevistado 4 & IES pública & Mestrado Profissional em Empreendedorismo \\
\hline
\end{tabular}

Future Studies Research Journal ISSN 2175-5825 SÃo PAUlo, v.11, N.3, P. 255 - 280, SeP. / DeC. 2019 
Entrevistado 5 IES pública

Programa de Pós-Graduação em Administração

Quadro 1: Peritos entrevistados

Fonte: Dados da pesquisa

Os mesmos foram selecionados mediante acessibilidade. E justifica-se a escolha destes peritos entrevistados também, em virtude destes serem todos professores de Programas de Pós-Graduação stricto sensu com experiência como docentes em mestrado/doutorado e como pesquisadores contemplando em seu bojo o foco da estratégia em várias nuances, dentre elas a prospecção de cenários.

Com estes cinco entrevistados iniciou-se a construção de uma lista de eventos (Brock \& Barry, 2003), pois, estes peritos são sabedores de informações úteis, de observações e impressões importantes para esses eventos futuros (Blanning \& Reinig, 2002). Dando continuidade à construção dos eventos, uma listagem destes eventos foi enviada, por meio de perguntas, utilizando uma survey a 35 peritos, conseguindo uma devolutiva de 29 (Quadro 2) para que indicassem a probabilidade de ocorrência destes eventos e o quão favorável e pertinentes estes serão caso ocorra em uma linha graduada de zero a dez. Ressalva-se que os dados foram coletados mediante uma survey online, hospedada na plataforma Survey Monkey e distribuída por diversas mídias para os 35 peritos. A survey foi conduzida de forma que o respondente deliberadamente respondia ao questionário.

\begin{tabular}{|c|c|c|c|}
\hline Peritos (P) & Titulação & Organização & Função \\
\hline P1 & Doutor(a) & IES privada & Diretor(a) \\
\hline P2 & Doutor(a) & IES pública & Docente/Pesquisador(a) \\
\hline P3 & Doutor(a) & IES privada & Docente/Pesquisador(a) \\
\hline P4 & Doutor(a) & IES privada & Docente/Pesquisador(a) \\
\hline P5 & Doutor(a) & IES privada & Docente/Pesquisador(a) \\
\hline P6 & Doutor(a) & IES pública & Docente/Pesquisador(a) \\
\hline P7 & Doutor(a) & IES privada & Docente/Pesquisador(a) \\
\hline P8 & Doutor(a) & IES privada & Docente/Pesquisador(a) \\
\hline P9 & Doutor(a) & IES pública & Docente/Pesquisador(a) \\
\hline P10 & Doutor(a) & IES pública & Docente/Pesquisador(a) \\
\hline P11 & Doutor(a) & IES pública & Docente/Pesquisador(a) \\
\hline P12 & Doutor(a) & IES privada & Diretor(a) \\
\hline P13 & Doutor(a) & IES privada & Docente/Pesquisador(a) \\
\hline P14 & Doutor(a) & IES privada & Diretor(a) \\
\hline P15 & Doutor(a) & IES privada & Docente/Pesquisador(a) \\
\hline P16 & Doutor(a) & IES pública & Docente/Pesquisador(a) \\
\hline P17 & Doutor(a) & IES pública & Docente/Pesquisador(a) \\
\hline P18 & Doutor(a) & IES pública & Docente/Pesquisador(a) \\
\hline P19 & Doutor(a) & IES pública & Docente/Pesquisador(a) \\
\hline P20 & Doutor(a) & IES pública & Docente/Pesquisador(a) \\
\hline P21 & Doutor(a) & IES privada & Docente/Pesquisador(a) \\
\hline
\end{tabular}


Henrique César Melo Ribeiro, Sergio Henrique Arruda Cavalcante Forte

\begin{tabular}{|c|c|c|c|}
\hline P22 & Doutor(a) & IES privada & Docente/Pesquisador(a) \\
\hline P23 & Doutor(a) & IES privada & Docente/Pesquisador(a) \\
\hline P24 & Doutor(a) & IES privada & Diretor(a) \\
\hline P25 & Doutor(a) & IES privada & Diretor(a) \\
\hline P26 & Doutor(a) & IES pública & Docente/Pesquisador(a) \\
\hline P27 & Doutor(a) & IES privada & Docente/Pesquisador(a) \\
\hline P28 & Doutor(a) & IES privada & Docente/Pesquisador(a) \\
\hline P29 & Doutor(a) & IES privada & Diretor(a) \\
\hline
\end{tabular}

Quadro 2: Peritos

Fonte: Dados da pesquisa

Aplicou-se o questionário de acordo com o método de sugerido por Blanning e Reinig (1998). Salienta-se que, no que tange a estrutura do questionário, foi embasado e norteado nos conceitos de pertinência (Marcial \& Grumbach, 2002) e de favorabilidade e probabilidade (Blanning \& Reinig, 1998). Com isso, em relação à pertinência, os peritos aferiram em uma escala tipo Likert de zero a cinco, em que (zero = sem influência; um = muito pouca influência; dois = pouca influência; três = razoável influência; quatro = forte influência; e cinco = extrema influência). De maneira geral, os peritos focaram sobre a pertinência da ocorrência ou não de algum evento na construção dos cenários, sendo o critério pertinência (importância) livre da possibilidade de ocorrência deste evento ou não (Marcial \& Grumbach, 2002).

No que se refere à favorabilidade, que versa sobre o quanto o evento é aderente para a construção dos cenários. Com isso, para esse critério, foi utilizada a escala (zero a 100\%, com intervalos de 10\% em 10\%). Para o discernimento da perspectiva de ocorrência das variáveis na construção dos cenários, também foi utilizada a escala de zero a 100\%, com intervalos de $10 \%$ em $10 \%$ (Blanning \& Reinig, 1998). Justifica-se que a escolha pela escala do tipo Likert se deve às seguintes vantagens: (i) fácil para o pesquisador construí-la e aplicá-la, assim como é simplificada para o respondente entendê-la e compreendê-la, tornando aceitável o breve retorno do respondente (Malhotra et al., 2005).

Logo em seguida, foi realizada a construção de uma matriz de eventos, colocando no eixo horizontal as expectativas de ocorrência destes eventos $(P)$ e, no eixo vertical, o quanto cada evento é apto ou contrário (F) para as instituições que estão sendo desenvolvidas as análises. Por último, a Figura 1 mostra a construção de três cenários: (i) cenário otimista; (ii) cenário realista; e (ii) cenário pessimista (Blanning \& Reinig, 1998). 
Ressalta-se que o processo de filtragem dos eventos foi feito por meio das seguintes ações: (i) cinco especialistas indicaram admissíveis e possíveis eventos; (ii) uma survey a 29 peritos; e (iii) busca na literatura científica destes eventos (Maccari et al., 2009; Maccari, Lima \& Riccio, 2009; Maccari, Riccio \& Martins, 2013; Moritz et al., 2013; Moritz, Nuner \& Pereira, 2008; Moritz \& Pereira, 2005; Sousa, Forte \& Oliveira, 2012). Foi feito uma consolidação destes eventos, evitando assim duplicidade e ou redundância destes, surgindo com isso os eventos evidenciados na Tabela 1.

Tabela 1: Eventos

\begin{tabular}{|c|c|c|c|}
\hline Eventos & $\begin{array}{l}\text { Perti } \\
\text { nênc } \\
\text { ia }\end{array}$ & $\begin{array}{l}\text { Favor } \\
\text { abilid } \\
\text { ade }\end{array}$ & $\begin{array}{l}\text { Proba } \\
\text { bilida } \\
\text { de }\end{array}$ \\
\hline $\begin{array}{l}\text { O programa procura manter as notas de excelência da Coordenação } \\
\text { de Aperfeiçoamento de Pessoal de Nível Superior (CAPES) }\end{array}$ & $14 \%$ & $\begin{array}{r}80,53 \\
\%\end{array}$ & $\begin{array}{r}77,37 \\
\%\end{array}$ \\
\hline $\begin{array}{l}\text { O programa incentiva a participação dos docentes em grupos de } \\
\text { pesquisa, convênios e intercâmbios com universidades e centros de } \\
\text { pesquisa do exterior }\end{array}$ & $17 \%$ & $\begin{array}{r}80,00 \\
\%\end{array}$ & $\begin{array}{r}66,32 \\
\%\end{array}$ \\
\hline $\begin{array}{l}\text { O programa incentiva a publicação em periódicos internacionais } \\
\text { como fator de impacto }\end{array}$ & $15 \%$ & $\begin{array}{r}80,00 \\
\% \\
\end{array}$ & $\begin{array}{r}73,68 \\
\% \\
\end{array}$ \\
\hline $\begin{array}{l}\text { Corpo docente de alta performance na produção acadêmica e } \\
\text { relevância internacional é recurso estratégico }\end{array}$ & $19 \%$ & $\begin{array}{r}78,95 \\
\%\end{array}$ & $\begin{array}{r}72,11 \\
\%\end{array}$ \\
\hline $\begin{array}{l}\text { Recursos estratégicos criam vantagem competitiva sustentável no } \\
\text { programa de pós-graduação stricto sensu }\end{array}$ & $14 \%$ & $\begin{array}{r}76,84 \\
\%\end{array}$ & $\begin{array}{r}67,89 \\
\% \\
\end{array}$ \\
\hline $\begin{array}{l}\text { Grupos e projetos de pesquisa são formados para fortalecer a } \\
\text { publicação científica, bem como parcerias internacionais }\end{array}$ & $18 \%$ & $\begin{array}{r}76,32 \\
\%\end{array}$ & $\begin{array}{r}70,00 \\
\%\end{array}$ \\
\hline $\begin{array}{l}\text { Recursos estratégicos são essenciais para qualidade no programa } \\
\text { de pós-graduação stricto sensu }\end{array}$ & $13 \%$ & $\begin{array}{r}75,26 \\
\%\end{array}$ & $\begin{array}{r}68,95 \\
\%\end{array}$ \\
\hline $\begin{array}{l}\text { A presença de renomados professores internacionais em eventos, } \\
\text { palestras, cursos, e "summercourses", incentivam a } \\
\text { internacionalização do programa }\end{array}$ & $20 \%$ & $\begin{array}{r}74,21 \\
\%\end{array}$ & $\begin{array}{r}66,84 \\
\%\end{array}$ \\
\hline $\begin{array}{l}\text { O programa incentiva, com recursos, a participação de docentes e } \\
\text { discente em eventos internacionais }\end{array}$ & $20 \%$ & $\begin{array}{r}73,16 \\
\%\end{array}$ & $\begin{array}{r}64,21 \\
\% \\
\end{array}$ \\
\hline $\begin{array}{l}\text { Recursos estratégicos impactam na formação de pesquisadores e } \\
\text { contribuem no desenvolvimento de suas atividades }\end{array}$ & $16 \%$ & $\begin{array}{r}73,16 \\
\%\end{array}$ & $\begin{array}{r}66,32 \\
\% \\
\end{array}$ \\
\hline $\begin{array}{l}\text { Recursos estratégicos são preponderantes para incentivar a } \\
\text { internacionalização do Programa de Pós-Graduação Stricto Sensu }\end{array}$ & $18 \%$ & $\begin{array}{r}72,63 \\
\%\end{array}$ & $\begin{array}{r}63,16 \\
\%\end{array}$ \\
\hline $\begin{array}{l}\text { O programa integra docentes de outras IESs para desenvolver } \\
\text { pesquisas conjuntas }\end{array}$ & $20 \%$ & $\begin{array}{r}72,11 \\
\%\end{array}$ & $\begin{array}{r}71,58 \\
\% \\
\end{array}$ \\
\hline $\begin{array}{l}\text { O processo seletivo estimula participação de candidatos, com base } \\
\text { no mérito e desempenho acadêmico }\end{array}$ & $20 \%$ & $\begin{array}{r}68,95 \\
\%\end{array}$ & $\begin{array}{r}66,84 \\
\% \\
\end{array}$ \\
\hline $\begin{array}{l}\text { O programa de Pós-Graduação Stricto Sensu influencia na atuação } \\
\text { de egressos em programas de stricto sensu de diferentes regiões }\end{array}$ & $19 \%$ & $\begin{array}{r}66,32 \\
\% \\
\end{array}$ & $\begin{array}{r}66,32 \\
\% \\
\end{array}$ \\
\hline
\end{tabular}

Fonte: Dados da pesquisa. 


\section{ANÁLISE E DISCUSSÃO DOS RESULTADOS}

Esta seção aborda a análise e a discussão dos resultados mediante as categorias: (I) cenários: otimista, realista e pessimista; e (II) descrição dos cenários otimista, realista e pessimista.

\subsection{CENÁRIOS: OTIMISTA, REALISTA E PESSIMISTA}

Entende-se que a prospecção de cenários força os gestores a pensar no futuro favorito (Wright \& Goodwin, 1999; Ithnin et al., 2018) e formular situações futuras (Moritz, Nuner \& Pereira, 2008), analisando decisões e o que poderia ser feito, e não o que foi realizado, possibilitando empreender diferentes unidades e modelos de negócios (Tapinos, 2013) para o futuro, alicerçado em uma busca criativa (Sousa, Forte \& Oliveira, 2012) de estratégias competitivas (Porter, 1980).

Por fim, têm-se a construção de três cenários, sendo que cada um abriga uma quantidade de eventos de acordo com sua probabilidade e favorabilidade (Spada \& Forte, 2018): (I) cenário otimista, que contempla os eventos com médias e altas expectativas de virem a ocorrer e que sejam mediana ou altamente adequadas para a área ou organizações; (II) cenário pessimista, que é constituído por eventos que apresentem médias e elevadas expectativas de virem a ocorrer e que sejam avessas para a área ou organizações; e (III) cenário realista, que conterá todos os eventos que proporcionem elevada perspectiva de virem se manifestar (Figueiredo, Chedid \& Machado Neto, 2010).

Em vista disso visualiza-se a Figura 1 que contempla a construção dos três cenários deste estudo: (i) cenário otimista; (ii) cenário pessimista; e (iii) cenário realista (Blanning \& Reinig, 1998). No que tange ao filtro de Pertinência, sua aferição foi alcançada por meio das seguintes etapas: (i) foi tirada a média pegando por base as respostas de Pertinência dos 29 peritos; (ii) foi calculado o desvio-padrão, também pegando por base as respostas de Pertinência dos 29 peritos; e (iii) foi mensurado o coeficiente de variação, por meio da divisão entre o desvio-padrão com a média.

Ressalta-se que os cenários são enquadrados em uma matriz com eixo horizontal e eixo vertical (Blanning \& Reinig, 1998; Heinzen, 2015). Em outras 
palavras, em um plano cartesiano, contemplado por eixos $X$ (versa a probabilidade) e $\mathrm{Y}$ (vislumbra a favorabilidade) (Spada \& Forte, 2018).

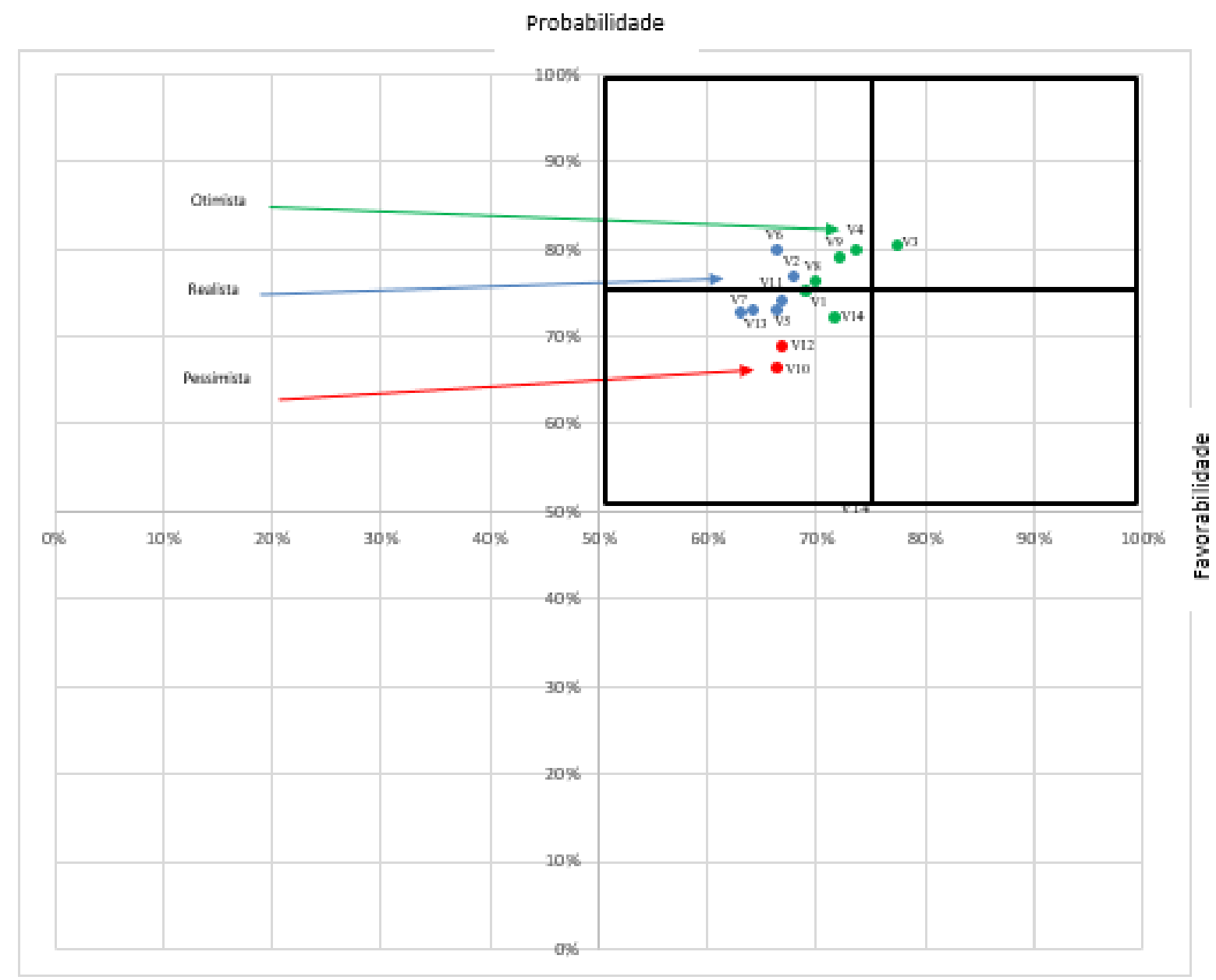

Figura 1: Cenários: otimista, realista e pessimista

Fonte: Dados da pesquisa

Legenda: V1 - Recursos estratégicos são essenciais para qualidade no programa de pós-graduação stricto sensu; V2 - Recursos estratégicos criam vantagem competitiva sustentável no programa de pós-graduação stricto sensu; V3 - O programa procura manter as notas de excelência da Coordenação de Aperfeiçoamento de Pessoal de Nível Superior (CAPES); V4 - O programa incentiva a publicação em periódicos internacionais como fator de impacto; V5 - Recursos estratégicos impactam na formação de pesquisadores e contribuem no desenvolvimento de suas atividades; V6 - O programa incentiva a participação dos docentes em grupos de pesquisa, convênios e intercâmbios com universidades e centros de pesquisa do exterior; V7 - Recursos estratégicos são preponderantes para incentivar a internacionalização do programa de pós-graduação stricto sensu; V8 - Grupos e projetos de pesquisa são formados para fortalecer a publicação científica, bem como parcerias internacionais; V9 - Corpo docente de alta performance na produção acadêmica e relevância internacional é recurso estratégico; V10 - O programa de pós-graduação stricto sensu influencia na atuação de egressos em programas de stricto sensu de diferentes regiões; V11 - A presença de renomados professores internacionais em eventos, palestras, cursos, e "summer courses" , incentivam a internacionalização do programa; V12 - O processo seletivo estimula participação de candidatos, com base no mérito e desempenho acadêmico; V13 - O programa incentiva com recursos a participação de docentes e discente em eventos internacionais; e V14 - O programa integra docentes de outras IESs para desenvolver pesquisas conjuntas.

\section{A Figura 1 é constituída e mostra os 14 eventos relevantes para a}

construção dos cenários (otimista, realista e pessimista), depurados assim: (i) no Future Studies Research Journal ISSN 2175-5825 São Paulo, v.11, N.3, P. 255 - 280, Sep. / Dec. 2019 
cenário otimista, seis eventos com médias e altas probabilidades de virem a correr e que são medianos ou altamente favoráveis para os programas stricto sensu ora analisados; (ii) no cenário realista, seis eventos que apresentam alta probabilidade de virem a se manifestar; e (iii) no cenário pessimista, dois eventos que apresentam médias e altas probabilidades de virem a acontecer e que são desfavoráveis para os programas objeto de estudo.

É importante salientar que alguns destes eventos estão abaixo da métrica, conforme preconiza a metodologia de Blanning e Reinig (1998), ou seja, considerados eventos de baixa ou média probabilidade e favorabilidade, mesmo assim podem ser considerados potencialmente importantes para os programas objeto de estudo.

\subsection{DESCRIÇÃO DOS CENÁRIOS OTIMISTA, REALISTA E PESSIMISTA}

Esta seção contempla a discussão dos três cenários e sobre os eventos que compõem cada um destes respectivamente para este estudo e análise agrupada dos referidos cenários.

\subsubsection{Eventos para o cenário otimista}

Observando a Figura 1, esta traz em seu bojo, seis eventos que fazem parte do cenário realista, são eles: (i) O Programa procura manter as notas de excelência da Coordenação de Aperfeiçoamento de Pessoal de Nível Superior (CAPES); (ii) O Programa incentiva a publicação em periódicos internacionais como fator de impacto; (iii) Corpo docente de alta performance na produção acadêmica e relevância internacional é recurso estratégico; (iv) Grupos e projetos de pesquisa são formados para fortalecer a publicação científica, bem como parcerias internacionais; (v) Recursos estratégicos são essenciais para qualidade no programa de pós-graduação stricto sensu; e (vi) O programa integra docentes de outras IES para desenvolver pesquisas conjuntas.

Isto posto, analisa-se e consta-se que o cenário otimista é constituído pelos eventos que proporcionam elevada probabilidade de ocorrência (Marcial \& Grumbach, 2002) e que são muito favoráveis para o Stricto Sensu das instituições 
de ensino superior públicas e privadas do Brasil da Área de Administração Pública e de Empresas, Ciências Contábeis e Turismo de 2019 a 2030.

Diante disso, realça-se que é importante que o programa procure manter ou alcançar as notas de excelência da Coordenação de Aperfeiçoamento de Pessoal de Nível Superior (CAPES), isso incentivará a publicação em periódicos internacionais com elevado fator de impacto (Moritz et al., 2013), subsidiado, alicerçado e norteado por um corpo docente de alta performance (CAPES, 2016) para efetivação da produção acadêmica de relevância internacional, sendo visto como recurso estratégico preponderante.

Ainda sobre o cenário otimista, neste contexto, vislumbram-se os grupos e projetos de pesquisas que são formados por estes pesquisadores, para melhor fortalecer as publicações científicas (CAPES, 2018), bem como por parcerias internacionais, impactando na qualidade do programa de pós-graduação stricto sensu (Maccari et al., 2009), criando vantagem competitiva (Cardoso \& Kato, 2015), e fazendo surgir recursos estratégicos valiosos, raros, de difícil ou demorada imitação e substituição, fazendo com que o programa torne-se bem gerido e competitivo no âmbito nacional e, consequentemente no enfoque internacional. Com isso, possibilitará que o programa possa ser reconhecido no contexto internacional, propiciando a integração de docentes de outras IES a posteriori para aperfeiçoar e fomentar pesquisas de qualidade em parceria (CAPES, 2018).

Os resultados aqui evidenciados são similarmente confirmados na pesquisa dos autores Moritz et al. (2013) que concluíram, em seu estudo, que é esperado pela pós-graduação para 2020 no Brasil, que ela aja com uma consciência social alargada, atenta ao ambiente para aprendizagens expressivas e aos novos tempos da humanidade, em que pesquisa e trabalho são variáveis que não se excluem, e os que nela atuam necessitam utilizar de suas competências para achar opções de modificação a partir de práticas inovadoras nos mestrados e doutorados, cujas diferentes demandas começam a colocar-se no presente, seja de fato relevante para um renovado e inovador Brasil do futuro. 


\subsubsection{Eventos para o cenário realista}

A Figura 1 também contempla em sua estrutura os seis eventos do cenário realista, são eles: (i) O Programa incentiva a participação dos docentes em grupos de pesquisa, convênios e intercâmbios com universidades e centros de pesquisa do exterior; (ii) Recursos estratégicos criam vantagem competitiva sustentável no programa de pós-graduação stricto sensu; (iii) A presença de renomados professores internacionais em eventos, palestras, cursos, e "summer courses" , incentivam a internacionalização do Programa; (vi) O Programa incentiva com recursos a participação de docentes e discente em eventos internacionais; (v) Recursos estratégicos impactam na formação de pesquisadores e contribuem no desenvolvimento de suas atividades; e (vi) Recursos estratégicos são preponderantes para incentivar a internacionalização do Programa de PósGraduação Stricto Sensu.

Já o cenário realista é formado por variáveis com grande probabilidade de acontecimentos (Moritz et al., 2013), podendo se mostrar como favoráveis para o Stricto Sensu das instituições de ensino superior públicas e privadas do Brasil da Área de Administração Pública e de Empresas, Ciências Contábeis e Turismo de 2019 a 2030. Isto posto, é preponderante que o Programa: estimule a participação dos docentes em grupos de pesquisa, convênios e intercâmbios com universidades e centros de pesquisa do exterior (CAPES, 2018), juntamente com a presença de renomados professores internacionais (Moritz et al., 2013), em eventos, palestras, cursos e summer courses, impactando na formação de pesquisadores e contribuindo no desenvolvimento de suas atividades.

Versa-se, também, a importância de o programa impulsionar com recursos estratégicos a participação de docentes e discentes em eventos internacionais, sendo preponderantes para incitar a estratégia de internacionalização do Programa de Pós-Graduação Stricto Sensu (CAPES, 2016; CAPES, 2018; Moritz et al., 2013), criando vantagem competitiva sustentável (Cardoso \& Kato, 2015) e valor acadêmico no âmbito nacional (Rieckmann, 2012).

Tais achados são analogamente corroborados pelas pesquisas de Alarcão et al. (2018) que construíram cenários prospectivos sobre a Universidade nas dimensões da formação, inovação e pesquisa e em função da intensidade valorativa. Os autores salientaram a oportunidade de melhor compreender um 
ambiente socioconstrutivo, refletindo sobre o que poderá ser a Universidade do futuro em uma visão a médio prazo e quais as implicações nas dimensões de formação, de inovação e de pesquisa; e Spada e Forte (2018) que identificaram os cenários prospectados para o horizonte do ano de 2030 para as Universidades Corporativas no Brasil. Concluíram que a prospecção de cenários traz às instituições de ensino superior brasileiras bases mais sólidas para compor suas estratégias em médio e longo prazos.

\subsubsection{Eventos para o cenário pessimista}

A Figura 1 ainda evidencia os dois eventos que são relacionados com o cenário pessimista, são eles: (i) O processo seletivo estimula participação de candidatos, com base no mérito e desempenho acadêmico; e (ii) O Programa de Pós-Graduação Stricto Sensu influencia na atuação de egressos em programas de stricto sensu de diferentes regiões.

Posto isto, manifesta-se que o cenário pessimista é composto por eventos que expõem médias e elevadas perspectivas de virem a ocorrer e que não são favoráveis (Figueiredo, Chedid \& Machado Neto, 2010; Spada \& Forte, 2018) para o Stricto Sensu das instituições de ensino superior públicas e privadas do Brasil da Área de Administração Pública e de Empresas, Ciências Contábeis e Turismo de 2019 a 2030.

Evidencia-se que a falta de um processo seletivo, que na não estimule a participação de candidatos com base no mérito e desempenho acadêmico, pode incorrer e influenciar negativamente na atuação de egressos em programas de stricto sensu de diferentes regiões do Programa de Pós-Graduação Stricto Sensu, inviabilizando o desenvolvimento regional.

\subsection{Análise agrupada dos três cenários}

Constata-se que os cenários prospectados neste estudo, definiram e estabeleceram eventos diversos (Saurin \& Ratcliffe, 2011) para os cenários otimista, realista e pessimista (Blanning \& Reinig, 1998) para os programas de pós-graduação stricto sensu da área de administração, ciências contábeis e 
turismo para o período de2019 a 2030, contribuindo para melhor entender e compreender a prospecção de cenários no ensino superior brasileiro, sobretudo no stricto sensu, trazendo, em seu bojo, alguns eventos que poderão ser importantes e necessários para emergir estratégias, que, de acordo com propósitos diferentes (Wilkinson, Kupers \& Mangalagiu, 2013), podendo assim ajudar tais programas em suas respectivas continuidades e na criação de vantagem competitiva no mercado (Faller \& Almeida, 2014).

\section{CONCLUSÃO}

O objetivo deste estudo foi elaborar os Cenários Prospectados para o período de 2019 a 2030 dos Programas de Stricto Sensu das Instituições do Brasil da Área de Administração Pública e de Empresas, Ciências Contábeis e Turismo. Para isso, utilizou-se a metodologia de cenários (Goodwin \& Wright, 2001), sugerida por Blanning e Reinig (1998).

Os achados assinalam 14 eventos proeminentes, seccionados como segue: seis eventos direcionados ao cenário otimista, seis eventos vinculados ao cenário realista e dois eventos conectados ao cenário pessimista, ou seja, aproximadamente $86 \%$ dos eventos fazem parte dos cenários otimista e realista. Tal achado é corroborado de maneira análoga na pesquisa de Spada e Forte (2018) que identificaram os cenários prospectados para o horizonte do ano de 2030 para as Universidades Corporativas no Brasil. Os achados apontaram 16 eventos relevantes sendo que a grande maioria dos eventos compõem os cenários otimista e realista, totalizando $93,75 \%$ e apenas $6,25 \%$ de eventos pessimistas.

No cenário realista, os eventos enfatizam a necessidade que os programas procuram ter no que tange a busca da excelência das notas para com a CAPES. Sendo que tal resultado e condição interage e relaciona-se com uma boa performance do corpo docente / pesquisadores, contribuindo também para alargar e robustecer a produção científica do programa, mediante publicações em periódicos com satisfatório fator de impacto. Sendo que este último, pode ser otimizado mediante a interação de grupos de estudos (parcerias com outros docentes e IES) por meio projetos de pesquisa e publicações simultaneamente. 
Já no cenário realista os eventos dão enfoque na participação dos professores em grupos de estudos internacionais. Tal ação, poderá possibilitar a vinda de docentes internacionais renomados para eventos, palestras, cursos, e "summer courses", contribuindo e incentivando na internacionalização do programa.

Porém, é importante que o programa estimule a presença de seus pesquisadores em eventos internacionais, influenciando em seu desenvolvimento e colaborando e impulsionando a internacionalização do programa, fato este que criará vantagem competitiva sustentável ao mesmo. Por fim, no cenário pessimista os eventos ponderam sobre a participação dos candidatos no processo seletivo por meio de seu mérito e desempenho acadêmico, vis-à-vis, caso os programas não se preocupem com tal ação, poderá influenciar na atuação de seus egressos em outros programas de stricto sensu de diferentes regiões do Brasil.

Conclui-se de maneira macro, uma visão sob a ótica de 14 eventos relevante categorizados nos cenários (otimista, realista e pessimista) elaborados e prospectados para os programas objeto de estudo. Tais cenários com seus respectivos eventos proeminentes dão um norte e alicerce a estes programas de como procederem em cenários adversos (Figueiredo, Chedid \& Machado Neto, 2010).

Isto posto, o referido estudo contribui para a literatura científica da área ao trazer em pauta eventos que poderão viabilizar iniciativas importantes para as IESs e seus respectivos programas de pós-graduação stricto sensu da área de administração, contabilidade e turismo em cenários de incertezas (Grant, 2003; Maggs \& Chelin, 2013) e cheios de desafios em decorrência da competitividade (Heinzen \& Marinho, 2019), perseguindo e alçando a posteriori melhores níveis de performance organizacional (Fernandes, Fleury \& Mills, 2006).

Como limitação do estudo, evidencia-se que se poderia ter tido mais repostas dos peritos, contudo, tal entrave não inviabilizou o referido estudo, visto que, tanto a questão como o objetivo foram respondidos e alcançados respectivamente. Como sugestões para estudos futuros, aconselha-se replicar o referido estudo, utilizando-se para isso outras áreas do conhecimento, contribuindo com isso para melhor entender e compreender como outros campos do saber se comportam na prospeç̧ão de cenários. 
Outra sugestão que se faz interessante e plausível de se evidenciar é, realizar de maneira comparativa, a prospecção de cenários da referida área com área análogo no âmbito internacional. Dando assim a possibilidade de observar e, consequentemente analisar como são os eventos e os cenários otimista, realista e pessimista comparativamente no contexto nacional e internacional.

Sugere-se também que dê continuidade no próprio estudo, no que se refere as estratégias que os programas stricto sensu utilizar-se-ão no período de 2019 a 2030, proporcionando assim saber, quais estratégias mais prováveis, por meio dos cenários prospectados para o referido período que os citados programas enfatizaram. E, por fim, realizar um estudo mediante clusters dos cenários otimista, realista e pessimista. Tais clusters podem ser das estratégias mais prováveis para cada cenário prospectado.

\section{REFERÊNCIAS}

Alarcão, I., Tavares, J., Mealha, Ó., \& Souza, D. N. de. (2018). Pensar a universidade dos próximos 20 anos através de uma metodologia de cenários. Revista Portuguesa de Educação, 31(1), 108-122.

Amer, M., Daim, T. U., \& Jetter, A. (2013). A review of scenario planning. Futures, 46, 23-40.

Bertero, C. O., Caldas, M. P., \& Wood Jr, T. (1999). Produção científica em administração de empresas: provocações, insinuações e contribuições para um debate local. Revista de Administração Contemporânea, 3(1), 147-178.

Blanning, R. W., \& Reinig, B. A. (1998). Building scenarios for Hong Kong using EMS. Long Range Planning, 31(6), 900-910.

Blanning, R. W., \& Reinig, B. A. (2002). Multiperiod scenario development using group support systems: an application to the future of Hong Kong. Journal of Organizational Computing and Electronic Commerce, 12(2), 105-119.

Blass, E., Thornton, M., \& Rawlings, B. (2011). Future scenarios for a depressed region of England: the role of education in creating alternative futures. Foresight, 13(6), 317.

Blois, H. D., \& Souza, J. C. (2008). Cenários prospectivos e a dinâmica de sistemas: proposta de um modelo para o setor calçadista. Revista de Administração de Empresas, 48(3), 35-45.

Bowman, G. (2016). The practice of scenario planning: an analysis of inter-and intraorganizational strategizing. British Journal of Management, 27(1), 77-96. 
Bradfield, R., Cairns, G., \& Wright, G. (2015). Teaching scenario analysis-An action learning pedagogy. Technological Forecasting and Social Change, 100, 44-52.

Brock, D. M., \& Barry, D. (2003). What if planning were really strategic? Exploring the strategy-planning relationship in multinationals. International Business Review, 12(5), 543-561.

Carlos, M. da G. de O., Paiva Filho, A. dos S., \& Forte, S. H. A. C. (2002). Contribuições recentes ao estudo de cenários na estratégia empresarial - miopia opcional ou tudo ainda embriões? Anais..., EnANPAD, 26. Salvador.

Chermack, T. J., Coons, L. M., Nimon, K., Bradley, P., \& Glick, M. B. (2015). The effects of scenario planning on participant perceptions of creative organizational climate. Journal of Leadership \& Organizational Studies, 21.

Cirani, C. B. S., Silva, H. H. M. da, \& Campanario, M. de A. (2012). A evolução do ensino da pós-graduação estrito senso em administração no Brasil. Revista de Administração Contemporânea, 16(6), 765-783.

Coordenação de Aperfeiçoamento de Pessoal de Nível Superior - CAPES. Documento de área: Administração Pública e de Empresas, Ciências Contábeis e Turismo. Recuperado em: <http://www.capes.gov.br/>.

Coordenação de Aperfeiçoamento de Pessoal de Nível Superior - CAPES. Proposta de aprimoramento do modelo de avaliação da PG: documento final da Comissão Nacional de Acompanhamento do PNPG 2011-2020 - 10/10/2018. Recuperado em: <file://C:/Users/hcmri/Downloads/PNPG\%20CS\%20Avaliac\%C3\%A30_Final_10\% 2010\%2018_CS_FINAL_17_55.pdf>.

Cardoso, A. L. J., \& Kato, H. T. (2015). Análise das publicações sobre capacidades dinâmicas entre 1992 e 2012: discussões sobre a evolução conceitual e as contribuições dos autores de maior notoriedade na área. Revista de Administração Mackenzie, 16(3), 201-237.

Crespi, T. B., Preusler, T. S., Luna, N. A., \& Ferreira, M. P. (2017). Novo Qualis: impacto na avaliação da produção intelectual dos pesquisadores em administração. Revista de Ciências da Administração, 19(47), 131-147.

Derbyshire, J. (2016). Potential surprise theory as a theoretical foundation for scenario planning. Technological Forecasting \& Social Change, 1-11.

Erfurth, A. E., \& Bezerra, F. A. (2013). Gerenciamento de resultados nos diferentes níveis de governança corporativa. Revista de Administração e Contabilidade da Unisinos, 10(1), 32-42.

Espinoza, F. da S., \& Hirano, A. S. (2003). As dimensões de avaliação dos atributos importantes na compra de condicionadores de ar: um estudo aplicado. Revista de Administração Contemporânea, 7(4), 97-117.

Faller, L. P., \& Almeida, M. I. R. de. (2014). Planejamento por cenários: preparando pequenas empresas do varejo de móveis planejados para um futuro competitivo. Revista de Administração da USP, 49(1), 171-187.

Fernandes, B. H. R., Fleury, M. T. L., \& Mills, J. (2006). Construindo o diálogo entre competência, recursos e desempenho organizacional. Revista de Administração de Empresas, 46(4), 48-65. 
Figueiredo, E. F., Chedid, S. E., \& Machado Neto, A. J. (2010). Cenários e orçamento empresarial: aplicação prática da metodologia de prospecção no Magazine Luiza. Anais... In: Encontro de Pesquisadores do Uni-FACEF - Centro Universitário de Franca, 11.

Godet, M. (2010). Future memories. Technological Forecasting and Social Change, 77(9), 1457-1463.

Godet, M. (1993). Manual de prospectiva estratégica: da antecipação à ação. Lisboa: Publicações Dom Quixote.

Goodwin, P., \& Wright, G. (2001). Enhancing strategy evaluation in scenario planning: a role for decision analysis. Journal of Management Studies, 38(1), 1-16.

Grant, R. M. (2003). Strategic planning in a turbulent environment: evidence from the oil majors. Strategic Management Journal, 24(6), 491-517.

Heinzen, D. A. de M. (2015). Alinhamento entre formulação e implementação da estratégia em Instituição de Ensino Superior, Tese (Doutorado em Administração e Turismo), Univalí, 202 pg.

Heinzen, D. A. de M., \& Marinho, S. V. (2019). Alinhamento entre formulação e implementação da estratégia em instituições de ensino superior no Brasil. Organizações em Contexto, 15(29), 279-322.

Höjer, M., Dreborg, K. H., Engström, R., Gunnarsson-Östling, U., \& Svenfelt, Á. (2011). Experiences of the development and use of scenarios for evaluating Swedish environmental quality objectives. Futures, 43, 498-512.

Ithnin, F., Sahib, S., Eng, C. K., Sidek, S., \& Harun, R. N. S. R. (2018). Mapping the futures of Malaysian higher education: a meta - analysis of futures studies in the Malaysian higher educations cenario. Journal of Futures Studies, 22(3), 1-18.

Lindgren, M., \& Bandhold, H. (2003). Scenario planning. Palgrave.

Maccari, E. A., Almeida, M. I. R. de, Nishimura, A. T., \& Rodrigues, L. C. (2009). A gestão dos programas de pós-graduação em administração com base no sistema de avaliação da Capes. Revista de Gestão USP, 16(4), 01-16.

Maccari, E. A., Lima, M. C., \& Riccio, E. L. (2009). Uso do Sistema de Avaliação da CAPES por Programas de Pós-Graduação em Administração no Brasil. Revista de Ciências da Administração, 11(25), 68-96.

Maccari, E. A., Riccio, E. L., \& Martins, C. B. (2013). A influência do sistema de avaliação da AACSB na gestão dos programas de pós-graduação stricto sensu em Administração nos Estados Unidos. Revista Eletrônica de Administração, 19(3), 738766.

Malhotra, N. K., Rocha, I., Laudisio, M. C., Altheman, É., Borges, F. M., \& Taylor, R. B. (2005). Introdução à pesquisa de marketing. São Paulo: Pearson Prentice Hall.

Marcial, E. C., \& Grumbach, R. J. S. (2002). Cenários prospectivos: como construir um futuro melhor. 4.ed. Rio de Janeiro: Editora FGV.

Moorcroft, S. (2015). Scenario based strategy - Navigate the future. Foresight, 17(5), 542-542. 
Moritz, G. de O., Moritz, M. O., Pereira, M. F., \& Maccari, E. A. (2013). A Pós-graduação brasileira: evolução e principais desafios no ambiente de cenários prospectivos. Future Studies Research Journal, 5(2), 03-34.

Moritz, G. de O., Nuner, R., \& Pereira, M. F. (2008). Os métodos de prospecção de cenários e sua aplicação nas organizações: um estudo de caso no período 1998-2008. Revista de Administração da FACES, 7(2), 68-83.

Moritz, G. de O., \& Pereira, M. F. (2005). Planejamento de cenários: a evolução do pensamento prospectivo. Revista Ciências da Administração, 7(13), 1-20.

Moro, A. B., Balsan, L. A. G., Costa, V. M. F., \& Schetinger, M. R. C. (2014). Clima organizacional: fatores significativos na percepção de docentes e discentes vinculados a programas de pós-graduação. Revista Gestão Universitária na América Latina, 7(3), 01-21.

Oliveira, A. S., Barros, M. D. de, Pereira, F. de C., Gomes, C. F. S., \& Costa, H. G. da. (2018). Prospective scenarios: a literature review on the scopus data base. Futures, $100,20-33$.

Oliveira, O., \& Forte, S. (2009). A indústria bancária brasileira: construindo cenários prospectivos e identificando as estratégias de utilização mais provável. Revista Portuguesa e Brasileira de Gestão, 64-77.

Oliveira, O. V. de, \& Forte, S. H. A. C. (2008). Banking industry: constructing prospective scenes and identifying strategies. Revista de Administração FACES, 7(3), 29-47.

Oliveira, O. V. de, \& Forte, S. H. A. C. (2011). Identificação dos recursos competitivos de utilização mais provável pela indústria bancária brasileira de 2008 a 2012. Revista de Administração da USP, 46(3), 243-257.

Paixão, R. B., \& Bruni, A. L. (2013). Mestrados profissionais: características, especificidades, diferenças e relatos de sucesso. Administração: Ensino e Pesquisa, 14(2), 279-309.

Patrus, R., Shigaki, H. B., \& Dantas, D. C. (2018). Quem não conhece seu passado está condenado a repeti-lo: distorções da avaliação da pós-graduação no Brasil à luz da história da Capes. Cadernos EBAPE. BR, 16(4), 642-655.

Pereira, F. de C., Barros, M. D. de, Oliveira, A. S., Gomes, C. F. S., \& Costa, H. G. (2015). Cenários prospectivos: estudo bibliométrico de artigos indexados na Base Scopus. Anais..., Encontro Nacional de Engenharia de Produção, 35, Fortaleza.

Porter, M. E. (1980). Competitive strategy: techniques for analyzing industries and competitors. New York: Free Press.

Ramirez, R., Mukherjee, M., Vezzoli, S., \& Kramer, A. M. (2015). Scenarios as a scholarly methodology to produce "interesting research". Futures, 71, 70-87.

Rieckmann, M. (2012). Future-oriented higher education: which key competencies should be fostered through university teaching and learning? Futures, 44(2), 127135.

Saurin, R., \& Ratcliffe, J. (2011). Using an adaptive scenarios approach to establish strategies for tomorrow's workplace. Foresight, 13(4), 46-63. 
Silva, A. T. B. da. (2010). Cenários do futuro e capacidades dinâmicas: um estudo Universidade de São Paulo (USP), FEA-USP, São Paulo.

Silva, M. C. da. (2016). Estratégias de criação de valor compartilhado à serem adotadas pela indústria da construção civil diante dos Cenários Prospectados para o período de 2016 a 2025. 2016. 185f. Dissertação (Mestrado em Administração de Empresas) Universidade de Fortaleza (UNIFOR), PPGA, Fortaleza.

Silva, M. C. da, Forte, S. H. A. C. (2016). Estratégias de criação de valor compartilhado a serem adotadas diante de cenários prospectados para a indústria da construção civil no Ceará. Future Studies Research Journal, 8(3), 227-254.

Silva, W. R., Machado Neto, A. J., \& Santos, L. A. (2015). Cenários prospectivos como ferramenta de gestão no ensino superior. Anais..., Congresso IFBAE, 8.

Sobreira, M. do C., Machado, C. O., Rebouças, C. Q., \& Forte, S. H. A. C. (2014). Cenários prospectivos no combate ao turismo sexual internacional em Fortaleza. Revista Turismo - Visão e Ação - Eletrônica, 16(2), 416-449.

Sousa, J. R. de, Forte, S. H. A. C., \& Oliveira, O. V. de. (2012). Recursos estratégicos no cenário 2009/2015 das IESS particulares da região nordeste do Brasil. Revista Ibero-Americana de Estratégia, 11(2), 91-119.

Spada, E., \& Forte, S. H. A. C. (2018). Cenários prospectivos das universidades corporativas no Brasil - 2030. Future Studies Research Journal, 10(2), 198-213.

Tapinos, E. (2013). Scenario planning at business unit level. Futures, 47, 17-27.

Thiesen, J. da S., Garcez, E. F., \& Guimaraes, J. E. (2014). Prospecção de cenários em educação: dos desafios do presente às possibilidades de futuro. Revista IberoAmericana de Estudos em Educação, 9(1), 1-17.

Thomas, J. R., Nelson, J. K., \& Silverman, S. J. (2007). Métodos de pesquisa em educação física. Porto Alegre: Editora ArteMed.

Varum, C. A., \& Melo, C. (2010). Directions in scenario planning literature-A review of the past decades. Futures, 42(4), 355-369.

Vecchiato, R. (2015). Strategic planning and organizational flexibility in turbulent environments. Foresight, 17(3), 257-273.

Weiss, L. A. S., Kremer, J. T., Trentin, E. T., \& Rojo, C. A. (2017). Cenários: uma análise da produção científica brasileira entre1980 e 2016. Future Studies Research Journal, 9(2), 116-139.

Wilkinson, A., Kupers, R., \& Mangalagiu, D. (2013). How plausibility-based scenario practices are grappling with complexity to appreciate and address 21st century challenges. Technological Forecasting and Social Change, 80(4), 699-710.

Wright, G., \& Goodwin, P. (2009). Decision making and planning under low levels of predictability: Enhancing the scenario method. International Journal of Forecasting, 25(4), 813-825. 